Original Research

\title{
A Study of Mental and Physiological Effects of Chronic Exposure to Noise in an Automotive Industry
}

\author{
Fakhradin Ahmadi Kanrash $^{1}{ }^{\mathrm{iD}}$, Iraj Alimohammadi ${ }^{{ }^{*}}{ }^{(\mathrm{D})}$, Jamileh Abolghasemi $^{3}{ }^{(\mathrm{D})}$ Kazem Rahmani $^{4}$
}

1. MSc. Student, Department of Occupational Health Engineering, Faculty of Health, Iran University of Medical Sciences, Tehran, Iran

2. Associate Professor, Department of Occupational Health Engineering, Faculty of Health, Iran University of Medical Sciences, Tehran, Iran

3. Assistant Professor, Department of Biostatistics, School of Public Health, Iran University of Medical Sciences, Tehran, Iran

4. MSc Student, Department of Epidemiology and Biostatistics, School of Public Health, Iran University of Medical Sciences, Tehran, Iran

\begin{tabular}{lr}
\hline \multicolumn{2}{c}{ Article Info } \\
\hline \multicolumn{2}{l}{ Original Article } \\
Received: $\quad 2019 / 01 / 20$ \\
Accepted: $\quad 2019 / 05 / 09$ \\
Published Online: & $2019 / 05 / 09$ \\
\multicolumn{2}{l}{ DOI: $10.30699 /$ jergon.7.1.54 }
\end{tabular}

Use your device to scan and read the article online

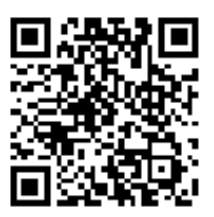

Corresponding Information

\section{Iraj Alimohammadi,} Associate Professor, Department of Occupational Health Engineering, Faculty of Health, Iran University of Medical Sciences, Tehran, Iran

Email:

irajrastin1@gmail.com

\begin{abstract}
Background and Objectives: Noise pollution is one of the most harmful physical factors in working environments in developed and developing countries, which has significant impacts on the workers' quality of life. The aim of this study was to investigate mental and physiological effects of chronic exposure to noise.
\end{abstract}

Methods: This is a cross-sectional study carried out randomly in 2018 on 250 workers in the automotive Industry. To measure the subjects' aggression and annoyance levels, the Buss and Perry's Aggression Questionnaire was used as well as an annoyance questionnaire. In this study, blood pressure measurement was also done using ALPK2 mercuric pressure gauge.

Results: The results showed that there was a significant difference in the systolic and diastolic blood pressure and noise annoyance in the groups with a lower-than- 85 and higher-than- $85 \mathrm{~dB}$ sound pressure levels $(P<0.001)$. Besides, the results of multivariate regression showed that the annoyance level and sound pressure level had a significant relationship with the workers' blood pressure $(P<0.01)$. Also, there was a significant correlation between aggression components and sound pressure level $(P<0.01)$.

Conclusion: The results of this study clearly showed that chronic exposure to noise in work environments was associated with psychological and physiological changes, such as increased blood pressure and aggression levels. Hence, implementation of preventive programs can help reduce the incidence of the psychological and physiological disorders.

Keywords: Occupational health, Aggression, Blood pressure, Mental health, Ergonomics

Copyright $($ C 2019, This is an original open-access article distributed under the terms of the Creative Commons Attribution-noncommercial 4.0 International License which permits copy and redistribute of the material just in noncommercial usages with proper citation.

How to Cite This Article:

Ahmadi Kanrash F, Alimohammad I, Abolaghasemi J, Rahmani K. A Study of Mental and Physiological Effects of Chronic Exposure to Noise in an Automotive Industry. Iran J Ergon. 2019; 7 (1) :54-62 


\title{
E-ISSN: 2345-5365 | مجلة اركونومى | سال \، شمارهٔ ا، بهار
}

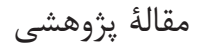 \\ بررسى اثرات روانى و فيزيولوزيكى ناشى از مواجهئه مزمن با صدا در \\ يك صنعت خودروسازى
}

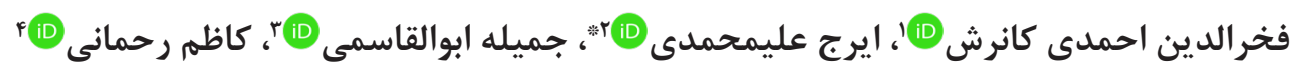

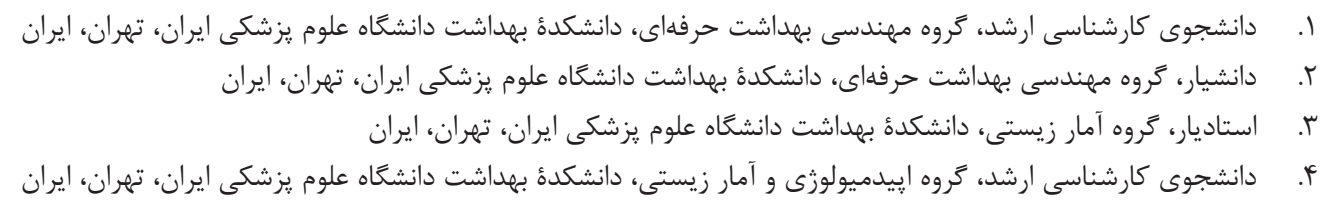

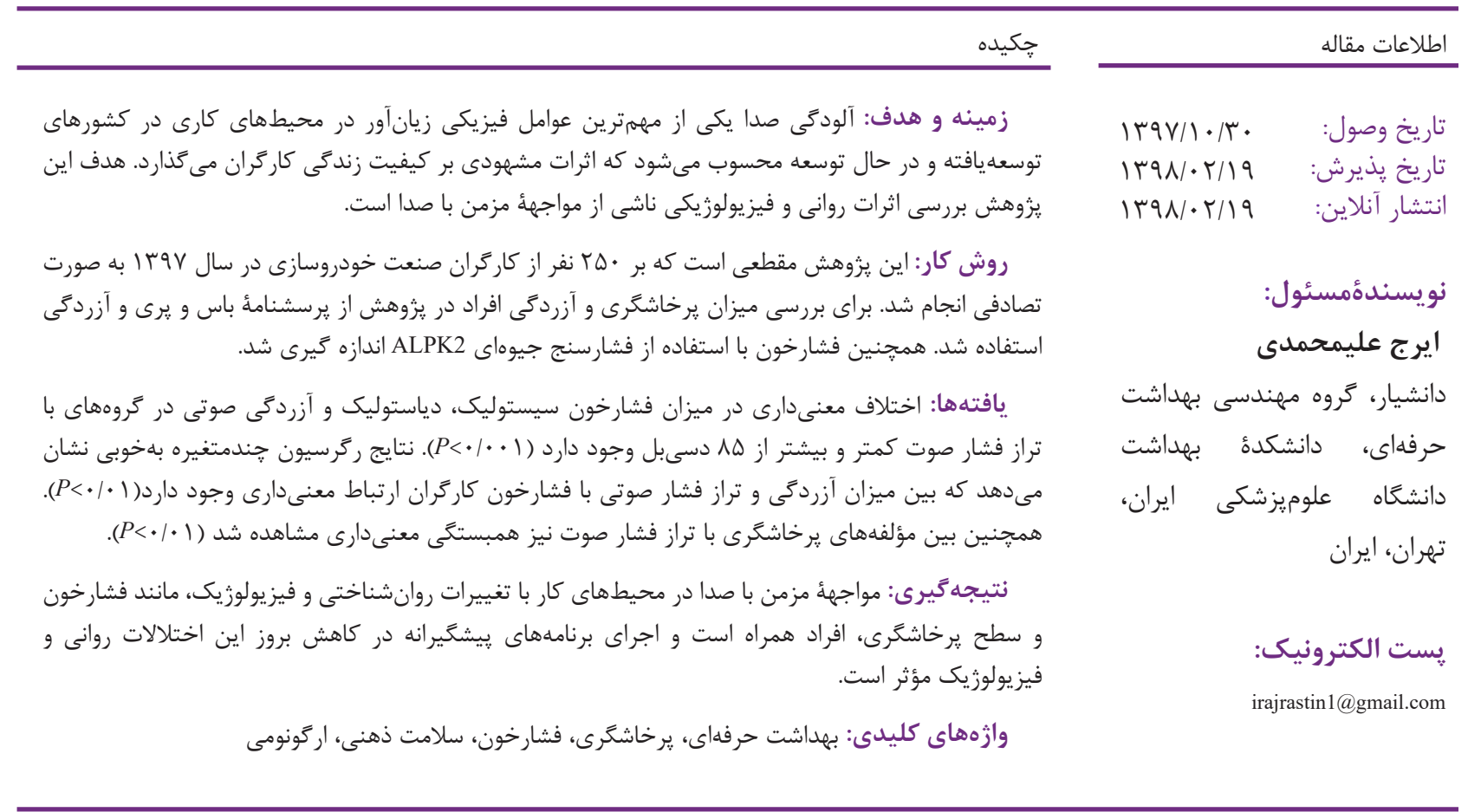

$[r, \Delta]$

مقلهم

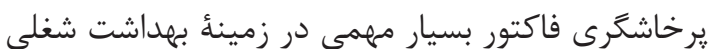

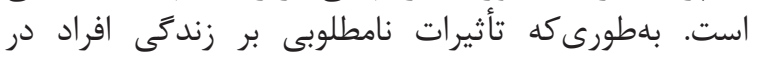

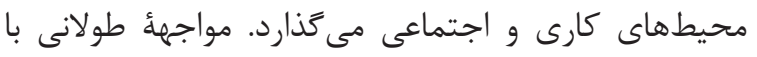

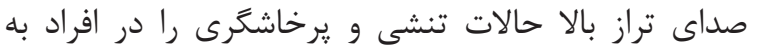

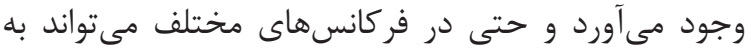

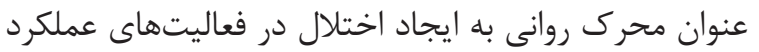

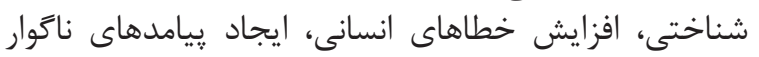

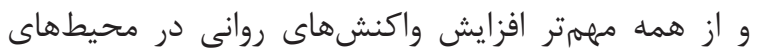

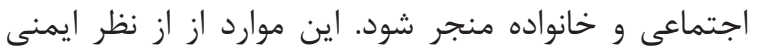

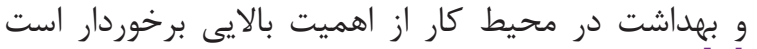

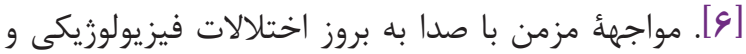

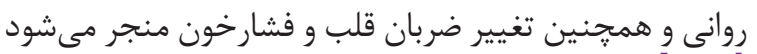
. $[v-9]$

مواجهه با صداى بالاتر از ه د دسىبل فشارخون سيستولى دئ

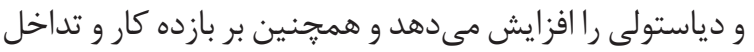

آلودگى صدا يكى از مهممترين عوامل فيزيكى زيانآور در محيطهاى كارى در كشورهاى توسعهيافته و در در حال توسئ فئه

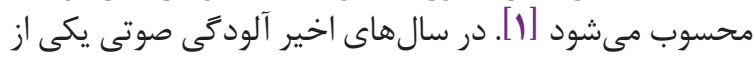

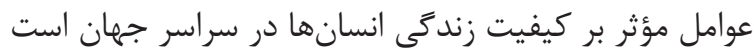

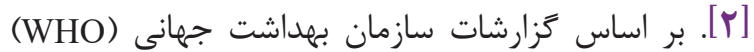

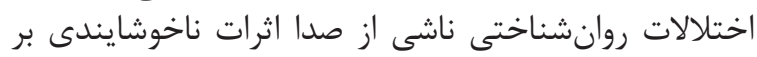

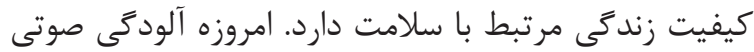

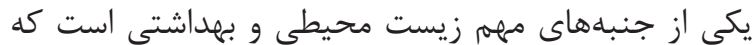

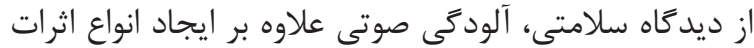

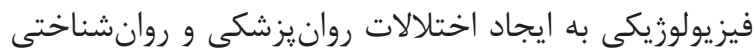

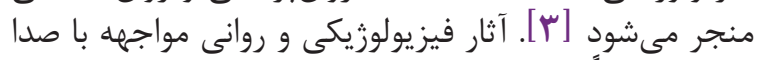

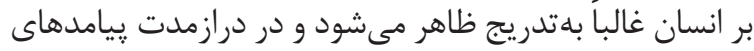

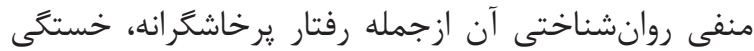

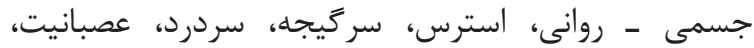
حواسيرتى، اختلال خواب، كاهش بازده كارى بروز ميجى كند 


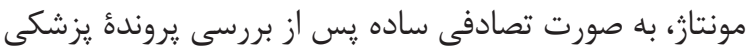

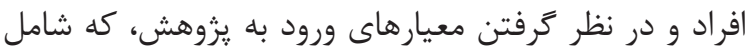

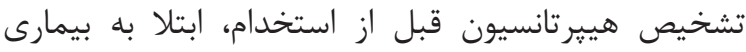

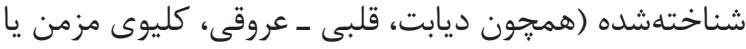

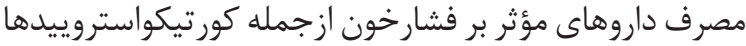

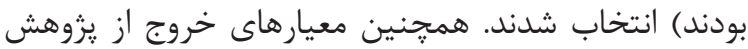

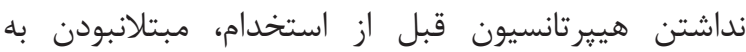

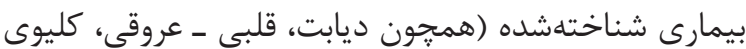

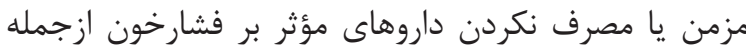

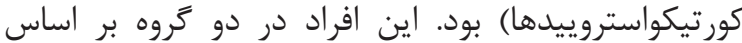

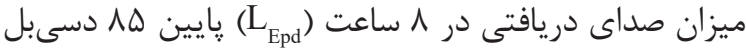

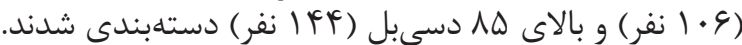
از سه ابزار براى انجام آزمايش استفاده شد:

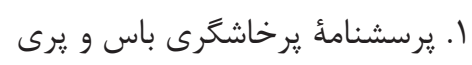

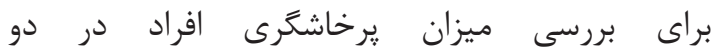

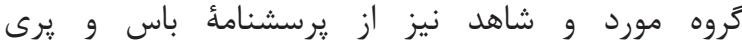
(The Aggression Questionnaire- AQ)

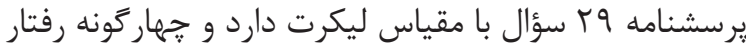

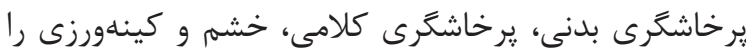

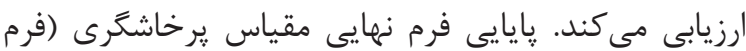

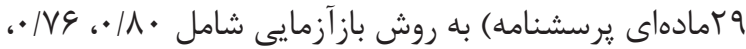

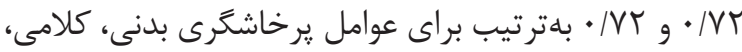

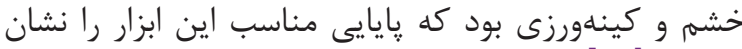

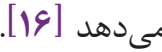

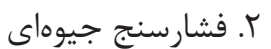

فشارخون با استفاده از فشارسنج جيوهاى ALPKY

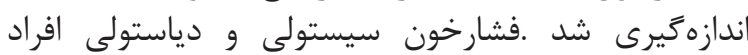

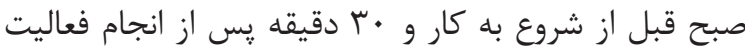

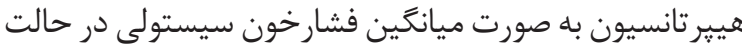

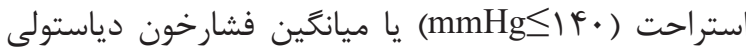

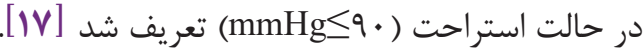

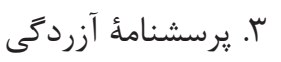

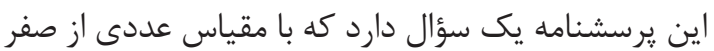

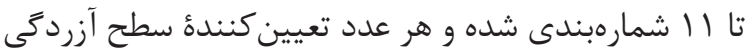

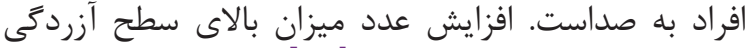

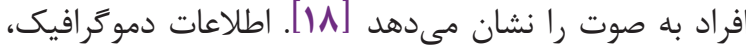

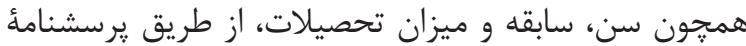

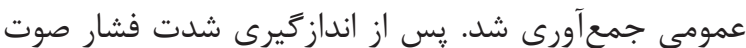

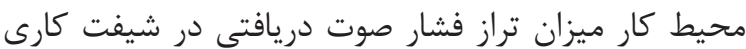

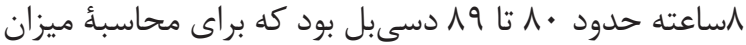

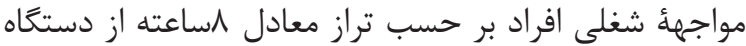

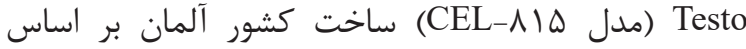

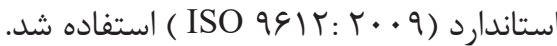

براى توصيف دادهها از جدول توزيع فراوانى و شاخصهاى

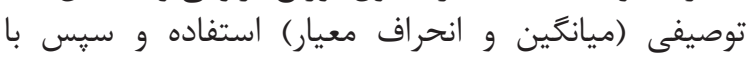

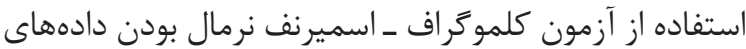

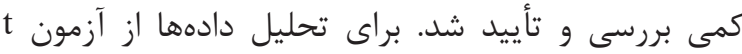

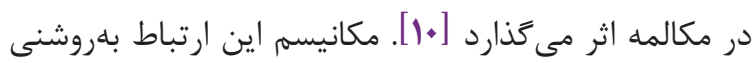

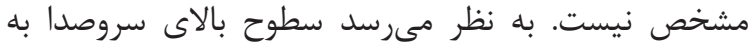

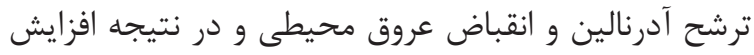

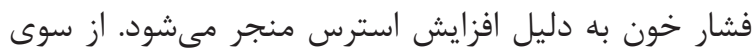

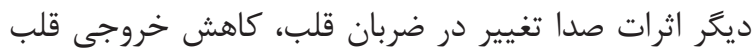

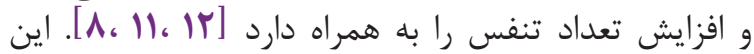

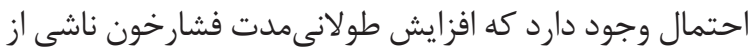

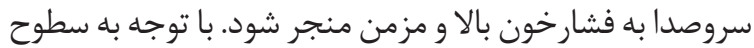

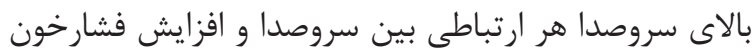

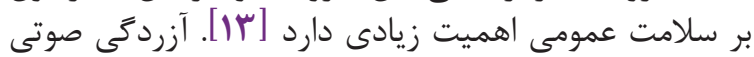

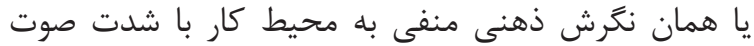

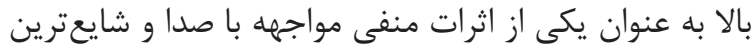

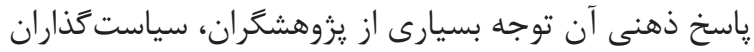

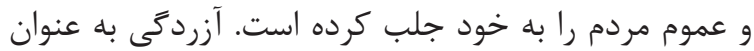

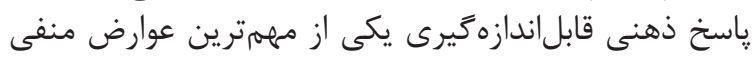

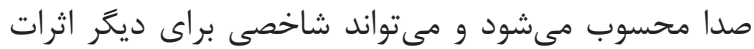

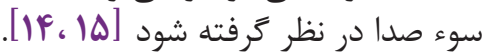
آزردكى به شرايط غيرمشخص اشاره دارد كه با مواردى إنى

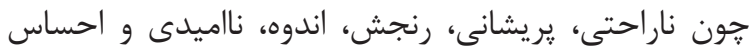

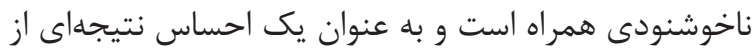

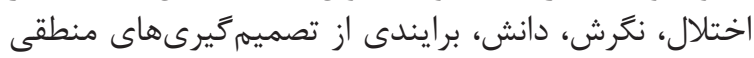

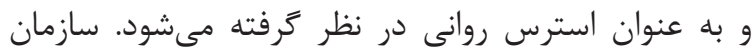

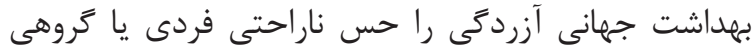

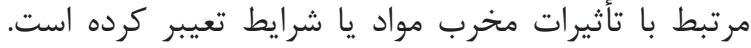

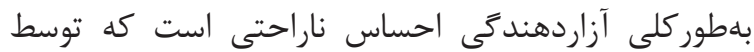

صداى ناخواسته و شرايط آن به وجود مي آيد [IF]

اين يزوهش باوجود اطلاعات كم به بررسى بيامدهاى صدا

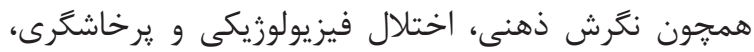

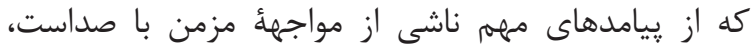

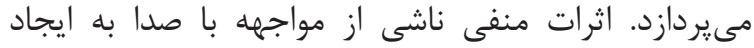

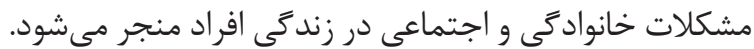

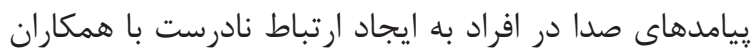

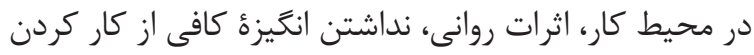

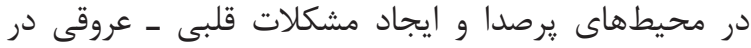

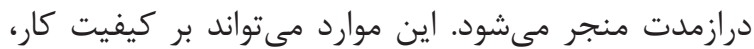

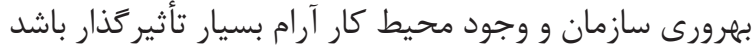

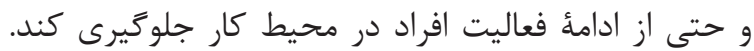

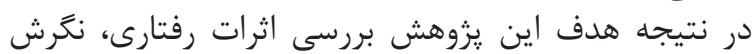

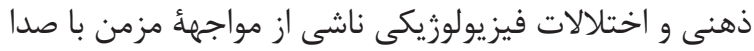

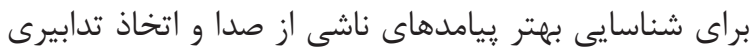

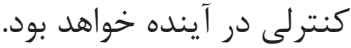

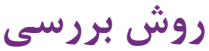

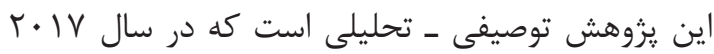

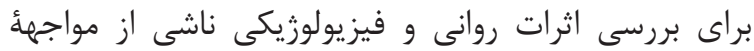

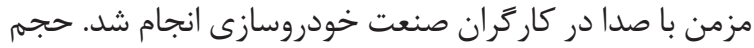

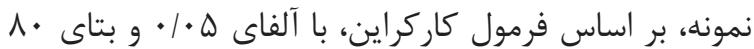

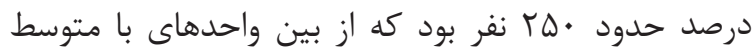

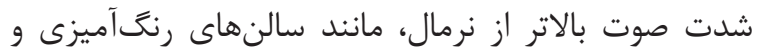




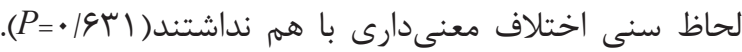

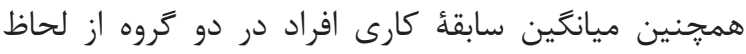

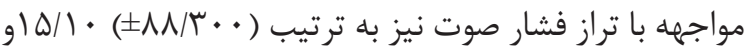

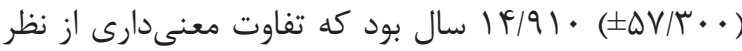

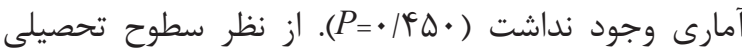

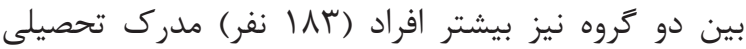

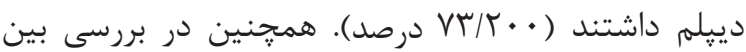

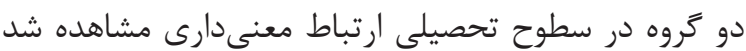
Nه

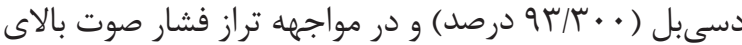

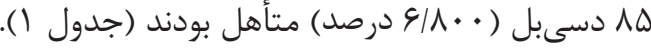
براى استفاده از آزمون t مستقل ارتباط بين فشارخون

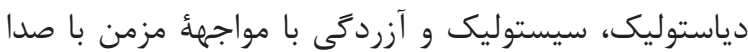

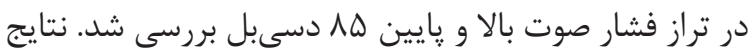

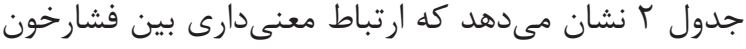

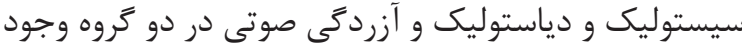

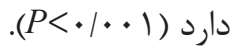

مستقل، براى بررسى اثر توام آزردگى صوتى و تراز فشار

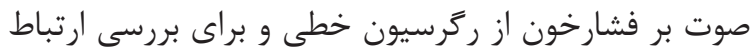

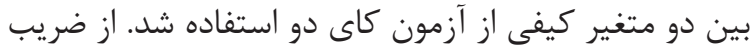

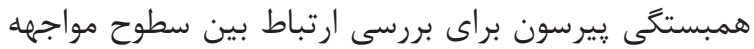

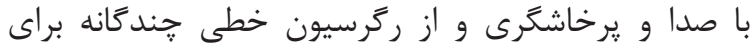

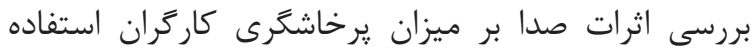

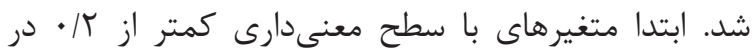

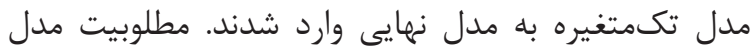

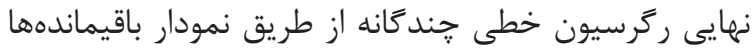

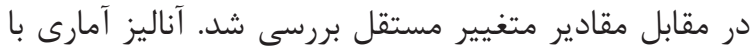

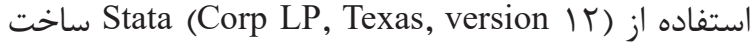
كشور امريكا انجام و سطح معنىدارى در اين يزول إنش كمتر

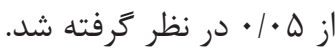

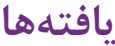

در اين يزوهش ميانكَين سنى افراد در دو كَروه مواجهه

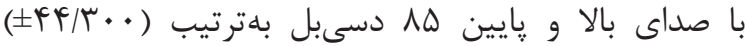

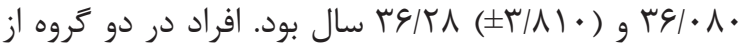

جدول ا. اطلاعات دموترافيك كارگران بر اساس مواجهه با صدا

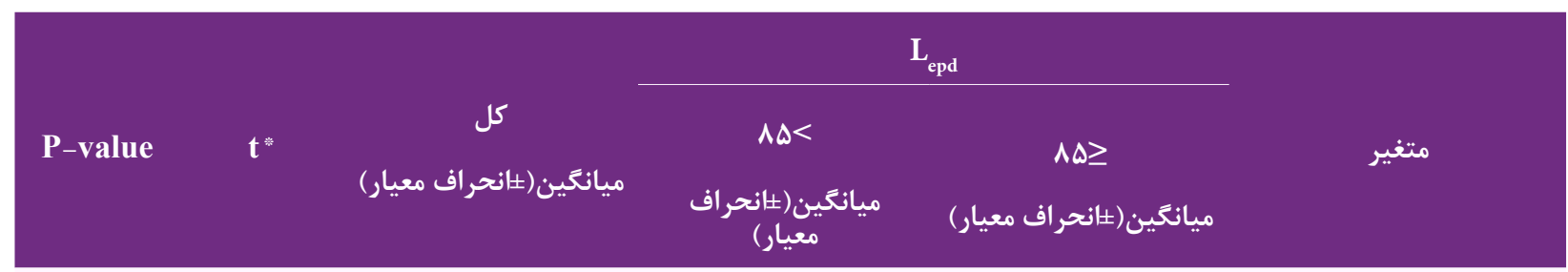

\begin{tabular}{|c|c|c|c|c|c|}
\hline . & $\cdot / \uparrow \wedge$ & $r G / / 9 \cdot( \pm r / V \Delta \cdot)$ & 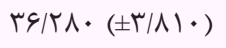 & $r \xi / \cdot G \cdot( \pm r / F \varphi \cdot)$ & سن \\
\hline$\cdot / 4 \Delta$ & $\cdot / V \Delta V$ & $|Q| \cdot T \cdot( \pm Y / \cdot Y \cdot)$ & $|\Delta /| \cdots( \pm r / \Lambda \Lambda \cdot)$ & $|F / q| \cdot( \pm r / \Delta V \cdot)$ & سابقه كار \\
\hline
\end{tabular}
P-value
$\mathrm{X}^{2}$
كل (\%)
تعداد (\%)
تعداد (\%)

\begin{tabular}{|c|c|c|c|c|c|c|}
\hline \multirow{3}{*}{.$/ \cdot r F$} & \multirow{3}{*}{ g/VF. } & IAK $(V T / Y \cdots)$ & $1 \cdot r(F, / r)$ & $\Lambda \cdot(r T)$ & دييلم & \multirow{3}{*}{ تحصيلات } \\
\hline & & $Q \cdot(T \cdot)$ & $r \Delta(\mid f)$ & $10(9)$ & فوق دييلم & \\
\hline & & $\operatorname{IV}(9 \mid \wedge \cdots)$ & $\varphi(r / T \cdots)$ & $\|(F / F \cdots)$ & كارشناسى به بالا & \\
\hline \multirow{2}{*}{ 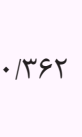 } & . /Ar. & $\mid V(\varepsilon \mid \wedge \cdots)$ & $\wedge(\Psi / \Gamma \cdots)$ & $9(\Gamma / 9 \cdot \cdots)$ & مجرد & \multirow{2}{*}{ وضعيت تأهل } \\
\hline & & rr (q/T) & $\mid r G(\Delta F / F \cdot)$. & q $(\Gamma \wedge / \Lambda \cdots)$ & متأهل & \\
\hline
\end{tabular}

*Statistically significant less than $<\cdot / \cdot \Delta$

**Statistically significant less than $<\cdot / \cdot$,

$$
\text { براى فشارخون سيستوليك و دياستوليك بهترتيب: }
$$

$\mathrm{SYS}=1 \cdot 9 / \uparrow \Delta q_{-1} \cdot \mid \Delta / r \mathrm{LEpd}+r / r \varepsilon \vee$ Annoyance

DIA $\left.=\Lambda r / 1 \cdot \vee-r / \Lambda) \cdot \operatorname{LEpd}_{+} \mid / \cdot \Delta\right)$ Annoyance

است. طبق اين دو معادله در صورت ثابت بودن تراز

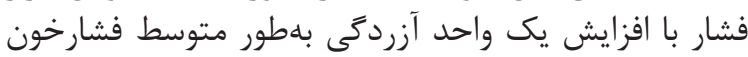

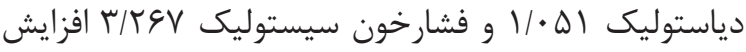

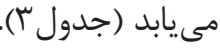

با استفاده از آزمون ركرسيون جند إنانه' ارتباط بين

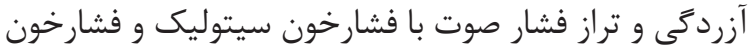

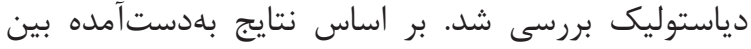

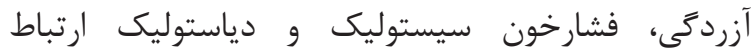

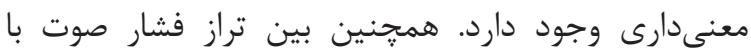

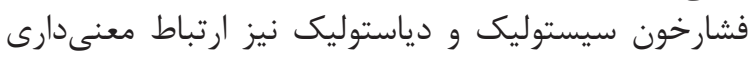

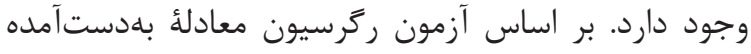

1. Multiple linear regression 
جدول ז. ارتباط بين فشارخون سيستوليك، دياستوليك با آزردَى صوتى و مواجهه با تراز فشار صوت با استفاده از آزمون t مستقل

\begin{tabular}{|c|c|c|c|c|}
\hline \multirow{3}{*}{$\mathbf{t}$} & \multirow{3}{*}{ P-value } & \multicolumn{2}{|c|}{$\mathbf{L}_{\text {epd }}$} & \multirow{3}{*}{ متغيرها } \\
\hline & & $\wedge \Delta<$ & $\wedge \Delta \geq$ & \\
\hline & & انحراف معيار \$ميانَين & انحراف معيار ذميانكين & \\
\hline 1./99. & $\cdot|\cdot|>$ & $|\kappa / r \cdots \pm| r \Delta / q \Lambda$. & $|\psi / \varnothing v \cdot \pm \|| \Delta / \Delta q$. & فشارخون سيستوليك \\
\hline $1 . / r r$. & $\cdot|\cdot .1\rangle$ & $r / q 4 \cdot \pm q \cdot / q r$. & $\Leftrightarrow / \mid \Delta \cdot \pm \Lambda r / \varphi \psi$. & فشارخون دياستوليك \\
\hline$|V| \cdot v \cdot$ & $\cdot|\cdot| 1>$ & $1 / 9 \mathrm{~V} \cdot \pm N / 1 \cdot$. & $1 / \Lambda r \cdot \pm \Delta / \cdot \Lambda \cdot$ & آزردَى \\
\hline
\end{tabular}

جدول ז. ارتباط بين آزردَى صوتى و تراز فشار صوت با فشارخون سيستوليك و دياستوليك با استفاده از ركرسيون جندمتغيره

\begin{tabular}{|c|c|c|c|c|c|c|}
\hline \multicolumn{2}{|c|}{$\mathbf{L}_{\text {epd }}$} & \multicolumn{2}{|c|}{ آزردَىى } & \multicolumn{2}{|c|}{ CONSTANT } & \multirow{2}{*}{ متغير ها } \\
\hline P-value & $\beta(\mathbf{s d})$ & P-value & $\beta(\mathbf{s d})$ & P-value & $\boldsymbol{\beta}(\mathbf{s d})$ & \\
\hline$\cdot|\cdot| 1\rangle$ & $r|\Lambda| \cdot(\cdot / 99 \cdot)$ & $\% \Delta$ & $\Gamma / \tau G V(\cdot / r \Delta \cdot)$ & $\cdot|\cdot| 1\rangle$ & $1 \cdot q / 4 q \Delta(r / q 4 \cdot)$ & فشارخون سيستوليك \\
\hline$\cdot|\cdot .1\rangle$ & $-1 \cdot|\Delta| r(\cdot / r \Delta \cdot)$ &.$/ .4$ & $1 / \cdot \Delta I(\cdot / r \varepsilon \cdot)$ & $\cdot|\cdot \cdot 1\rangle$ & $\Lambda T / 1 \cdot V(1 / V T r)$ & فشارخون دياستوليك \\
\hline
\end{tabular}

نتاج حاصل از ركرسيون خطى (جدول ه) نشان مى مهدي

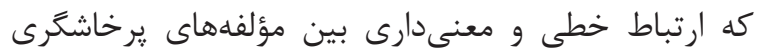

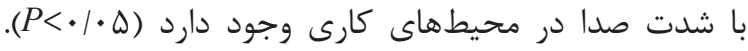

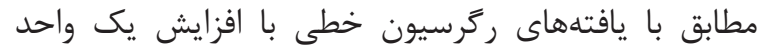

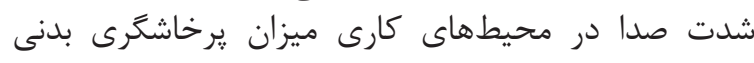

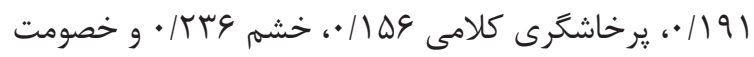

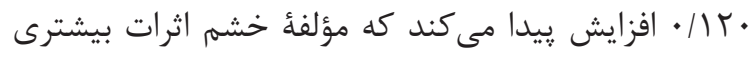

به ساير مؤلفهها دارد.

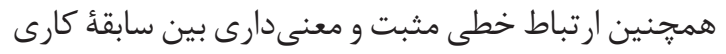

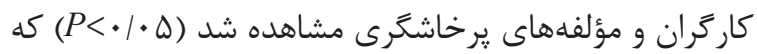

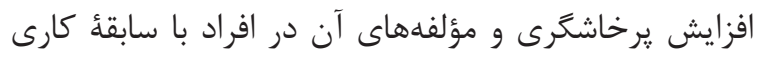

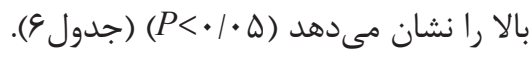

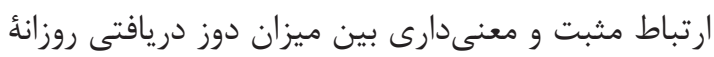

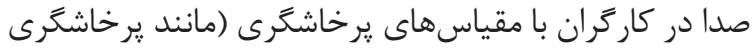

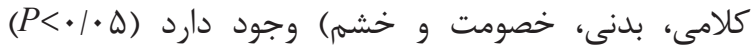

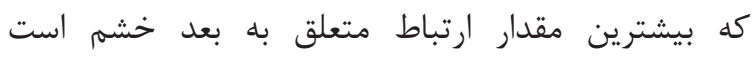

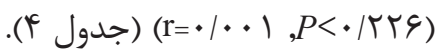

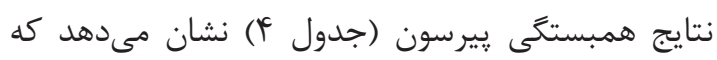

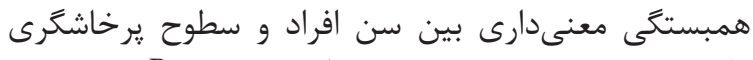

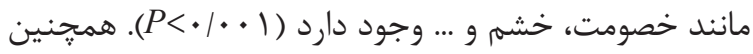

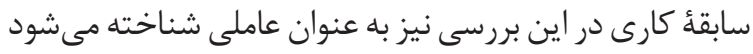

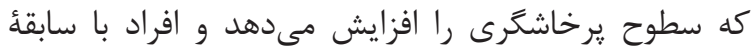

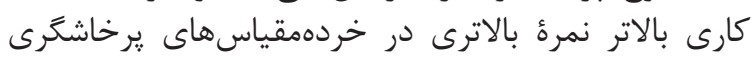

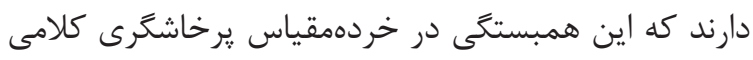

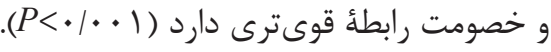

جدول F. همبستكى بين مقياسهاى يرخاشگرى با ميزان شدت دوز دريافتى صدا و ساير مؤلفهها در كاركران

\begin{tabular}{|c|c|c|c|c|c|c|}
\hline يرخاشَّى كل & خصومت & خشم & يرخاشَّى بدنى & يرخاشَرى كلامى & \multicolumn{2}{|c|}{ مقياس } \\
\hline .1119 &.$/ 149$ & . TTE & .119. & $.|| r \mid$ & $\mathrm{R}$ & \\
\hline$* 1 \cdot 1$ & $\cdot / \cdot r$. & $* / \cdot 1$ & $\cdot / \cdot 1$ & $\cdot / \cdot r$. & P-value & pd \\
\hline$\cdot / 4 \cdot 1$ & - Rat & $\cdot / r \wedge \Lambda$ & $\cdot|f| V$ &.$/ 4 \& V$ & $\mathrm{R}$ & \\
\hline$m \cdot \mid \cdot .1$ & 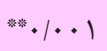 & $m \cdot 1 \cdots 1$ & $* . / \cdots 1$ & $*|| 1$. & P-value & سل \\
\hline . /rqf & & $\cdot / I V F$ & . & $\cdot|4| q$ & $\mathrm{R}$ & سابقهُ \\
\hline$* / . \cdot 1$ & 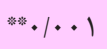 & $* \cdot \cdots 1$ & $* \cdot \cdot \cdot 1$ & $*|\cdot|$ & $\mathrm{P}$-value & كارى \\
\hline
\end{tabular}

*Statistically significant less than $<\cdot / \cdot 0$

$* *$ Statistically significant less than $<\cdot / \cdot 1$ 
جدول ه. ارتباط بين مؤلفههاى پيرخاشعرى كارَّران با ميزان و شدت صدا در محيط كار

\begin{tabular}{|c|c|c|c|c|}
\hline P-value & $\% ৭ \Delta$ confidence interval & Standard Error & Coefficient & متغير \\
\hline$\cdot .1 \cdot 11$ & (1) & $\cdot / \cdot v f$ & .1191 & يرخاشكرى بدنى \\
\hline.$/ .49$ & $\cdot / \cdot \Lambda_{-} \cdot r \cdot r$ & $\cdot / \cdot V \Delta$ & $\cdot / 1 \Delta \varphi$ & يرخاشكرى كلامى \\
\hline$\cdot 1 \cdot \cdot 1$ & س & $.1 \cdot 94$ & . & خشمى \\
\hline$\cdot / \cdot r \Lambda$ & $\cdot / \cdot I r-\cdot / 4 t \Lambda$ & $\cdot / \cdot \Delta f$ &.$/ 1 r$ & خصومت \\
\hline$=r$ & $\cdot 1 \cdot-\cdot r \cdot 1 \cdot q r$ & $\cdot / \cdot 11$ & $\cdot / \cdot \Delta V$ & يرخاشكرى كل \\
\hline
\end{tabular}

*Statistically significant less than $<\cdot / \cdot \Delta$

$* *$ Statistically significant less than $<\cdot / \cdot$,

جدول \&. ارتباط بين مؤلفههاى يرخاشگرى در كاركران با ميزان سابقهُ كارى

\begin{tabular}{|c|c|c|c|c|}
\hline P-value & $\% १ \Delta$ confidence interval & Standard Error & Coefficient & متغير \\
\hline$* \cdot \cdot \cdot 1$ & $\cdot / \cdot 19-\cdot / r \mu \Lambda$ & . I He & $\cdot / T \& 4$ & يرخاشگرى بدنى \\
\hline$\cdot 1 \cdot 1$ & $\cdot / I V V_{-} \cdot /$ TYY & $\cdot / \cdot r V$ & $\cdot / r \Delta \cdot$ & برخاشكَى كلامى \\
\hline$* 1 \cdot .9$ & $\cdot\left|\cdot r \varepsilon_{-} \cdot / r\right| \varepsilon$ & $\cdot 1 \cdot+\Delta$ &.$/ 1 T 4$ & خشم \\
\hline$\cdot \cdot 1 \cdot \cdot 1$ & $\cdot$ MFY-. $/ F Y G$ & $\cdot 1 \cdot \Delta 1$ & - MFD & 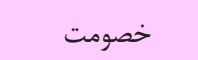 \\
\hline$\cdot 1 \cdot \cdot 1$ & $\cdot / V \cdot \cdot-1 / T V \cdot$ &.$/ 1 \notin 9$ &.$/ 919$ & يرخاشكرى كل \\
\hline
\end{tabular}

*Statistically significant less than $<\cdot / \cdot \Delta$

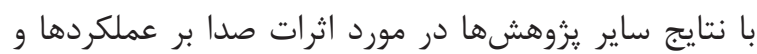

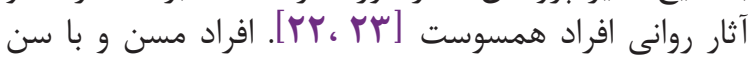
بالاتر كه با صداهاى مزمن در محيطهاى كارى هاى و غيركارى

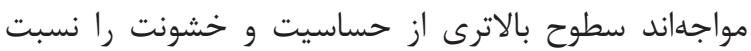

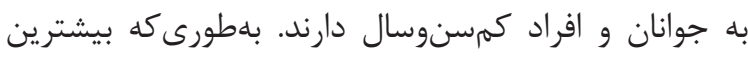

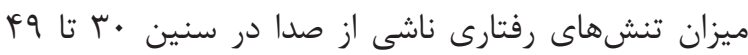

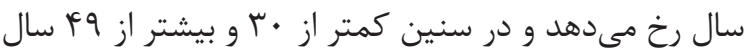

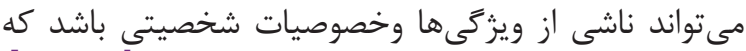

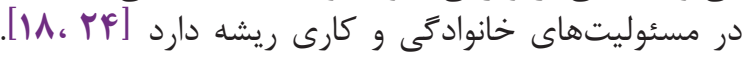

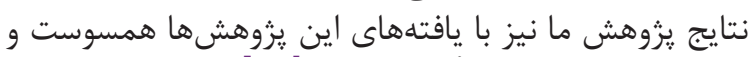

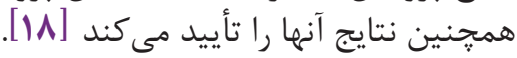

يكى از اثرات احتمالى صداى بلند به صورت مزمن، كه

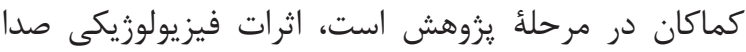

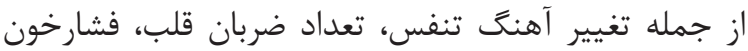

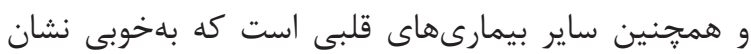

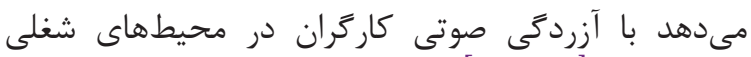

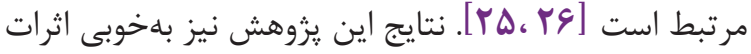

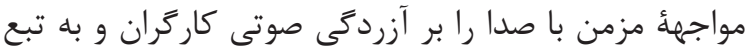

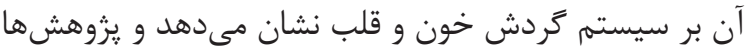

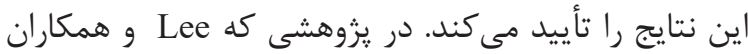

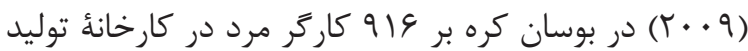

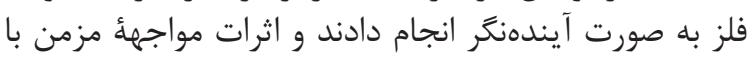

\section{بحث}

نتايج اين :روهش بهخوبى ارتباط بين سطوح و شدت

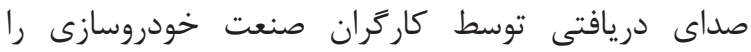

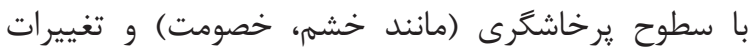

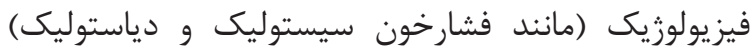
نشان مى ندهد.

خشونت و يرخاشخرى با سلامتى و رفتارهاى نامطلوب

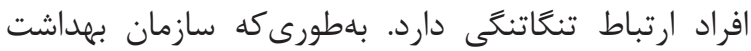

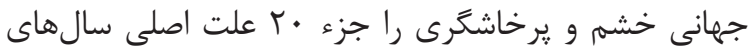
ازدسترفتهٔ عمر با ناتوانى (Disabled Age Lost Years)

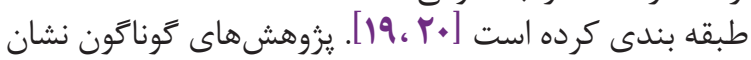

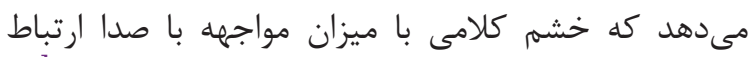

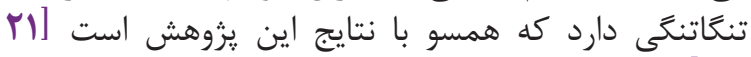

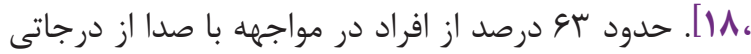

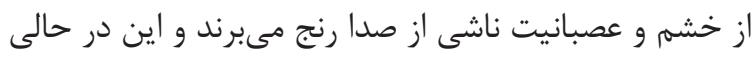

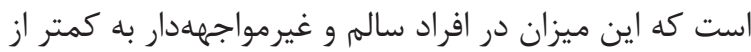

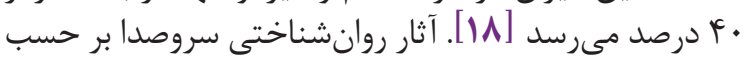

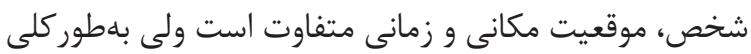

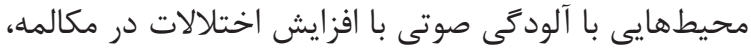

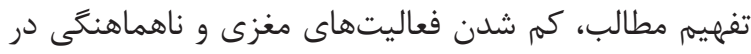

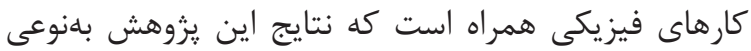


خود بهخوبى نتيجه كرفت كه ارتباط مثبت و معنى إدارى

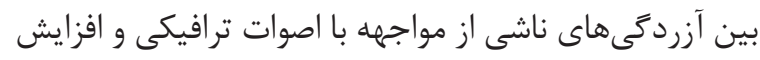

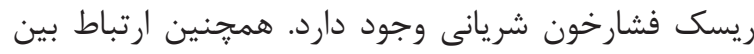

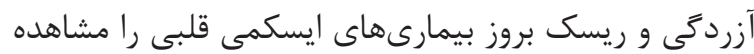

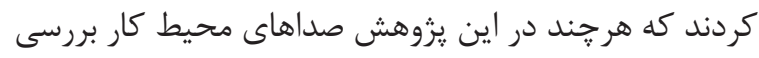

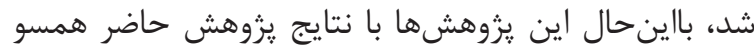

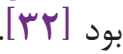

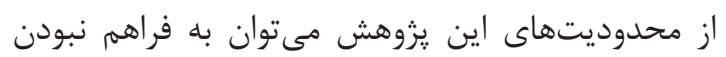

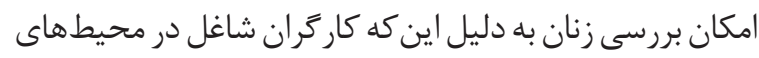

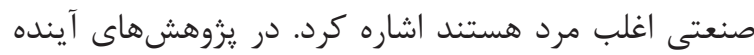

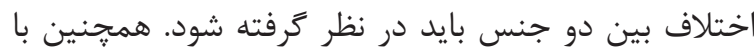

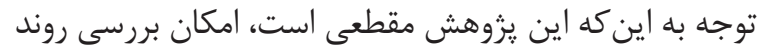

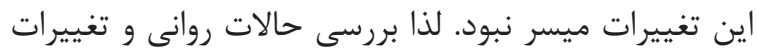

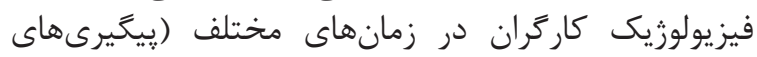
بعدى) نيز در درك بهتر اين ارتباط كمك دكنينده است.

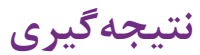

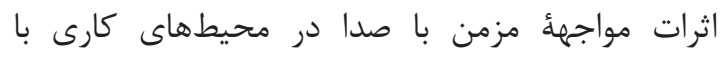

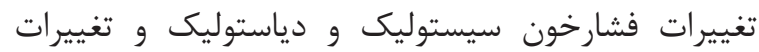

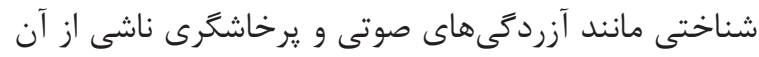

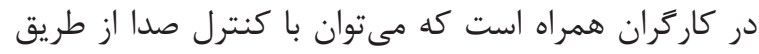

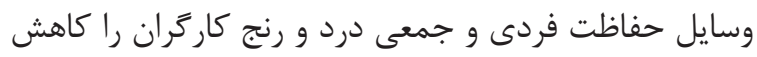
و كيفيت زندكى آنها را افزايش داد.

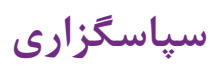

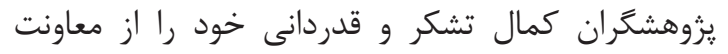

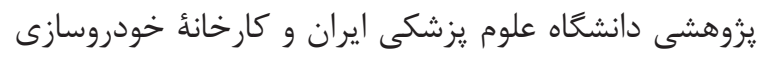

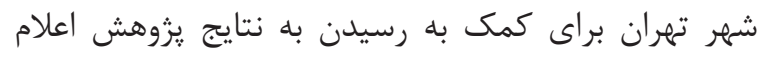
مى كنند.

$$
\text { تعارض در منافع }
$$

بين نويسند ًان هيجَّونه تعارضى در منافع وجود ندارد.

\section{References}

1. Fiedler PE, Zannin PH. Evaluation of noise pollution in urban traffic hubs-Noise maps and measurements. Environmental Impact Assessment Review. 2015 Feb 1;51:1-9.

2. Hunashal RB, Patil YB. Assessment of noise pollution indices in the city of Kolhapur, India. Procedia-Social and Behavioral Sciences. 2012 Jan 1;37:448-57.

3. Frick K. Work environment dialogue in a Swedish municipality-strengths and limits of the Nordic work environment model. Nordic journal of working life studies. $2013 \mathrm{Feb}$ 1;3(1):69.

4. Waye KP, Öhrström E. Psycho-acoustic characters of relevance for annoyance of wind turbine noise. Journal of sound and vibration. 2002 Feb 7;250(1):65-73.
سروصدا بر فشارخون را بررسى كردند ميانگين فشارخون

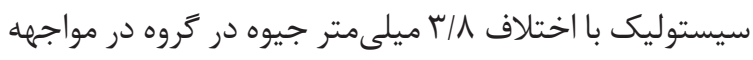

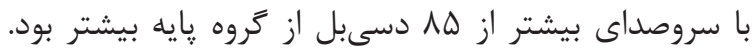

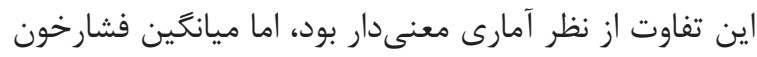

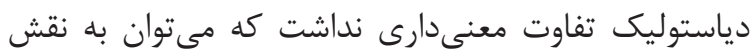

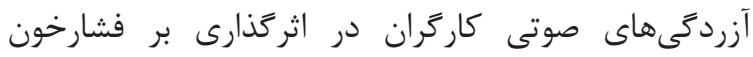

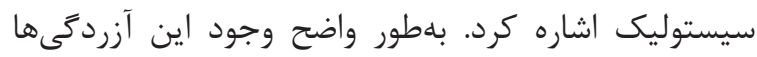

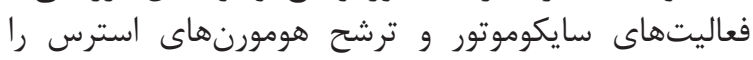

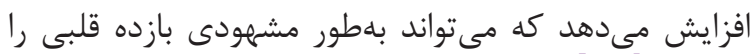

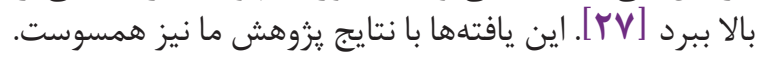

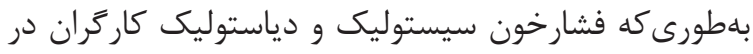

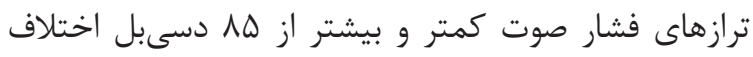

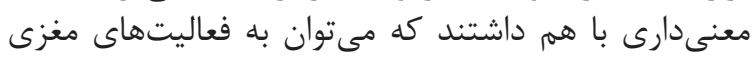

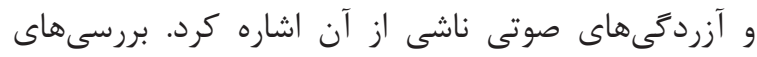

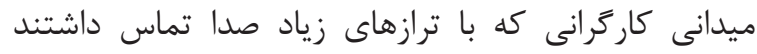

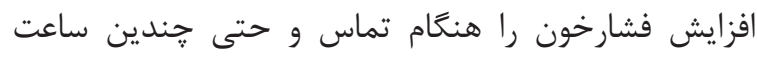

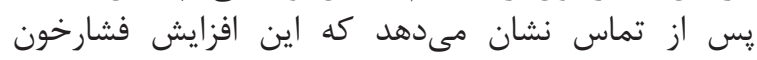

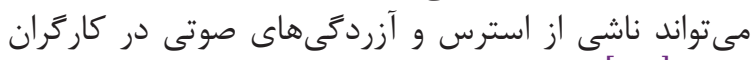

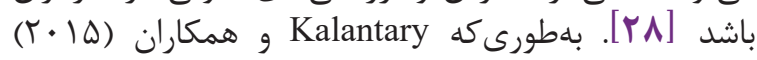

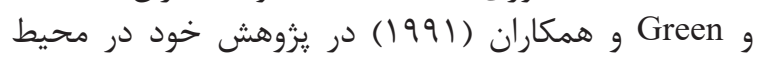

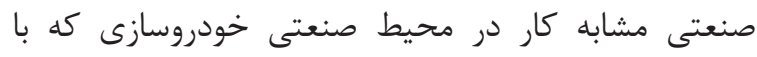

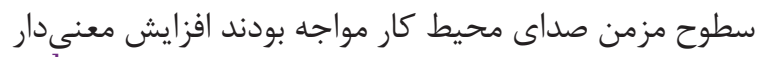

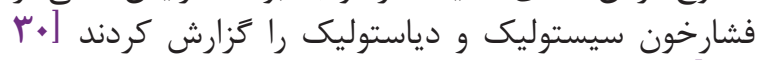

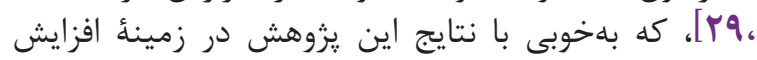

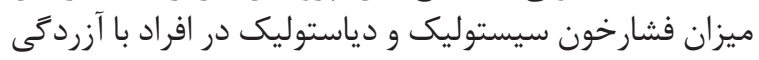

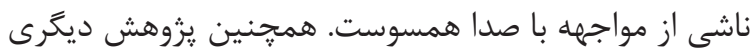

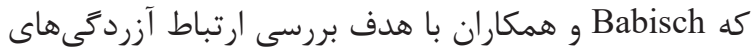

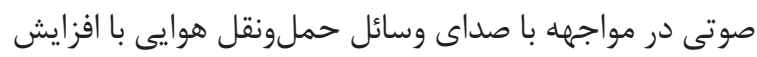

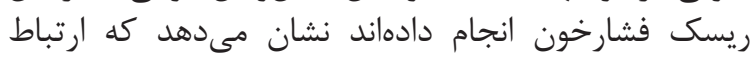

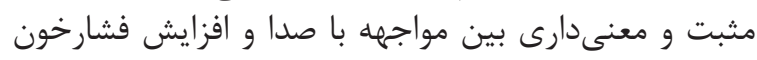

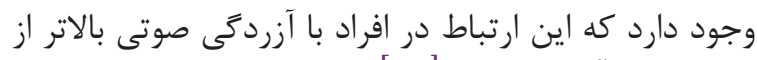

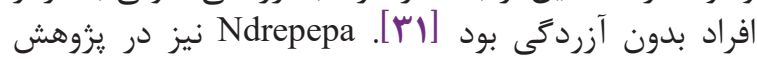

5. Pawlaczyk-Łuszczyńska MA, Dudarewicz AD, Waszkowska MA, Szymczak W, Śliwińska-Kowalska MA. The impact of low frequency noise on human mental performance. Inter $\mathrm{J}$ Occup Med Environ Health. 2005;18(2):18598.

6. Korte C, Grant R. Traffic noise, environmental awareness, and pedestrian behavior. Environment and Behavior. 1980 Sep;12(3):408-20.

7. Smith A. A review of the non-auditory effects of noise on health. Work \& stress. 1991 Jan 1;5(1):49-62.

8. Van Kempen EE, Kruize H, Boshuizen HC, Ameling CB, Staatsen BA, de Hollander AE. The association between noise exposure and blood pressure and ischemic heart disease: a meta-analysis. Environmental health perspec- 


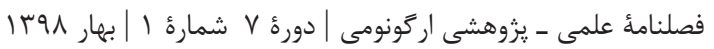

tives. 2002 Mar;110(3):307-17.

9. Alimohammad I, Salimi F, Rahmani K, Soltani R, Ahmadi Kanrash F. Relationship between Smoking and Hearing Impairment of Automotive Industry Workers Exposed to Noise. OCCUPATIONAL MEDICINE Quarterly Journal. 2019;10(3):31-9.

10. Motamedzade M, Ghazaiee S. Combined effects of noise and shift work on workers' physiological parameters in a chemical industry. 2003.

11. Chang TY, Jain RM, Wang CS, Chan CC. Effects of occupational noise exposure on blood pressure. Journal of occupational and environmental medicine. 2003 Dec 1;45(12):1289-96.

12. Pourabdiyan S, Ghotbi M, Yousefi HA, Habibi E, Zare MO. The epidemiologic study on hearing standard threshold shift using audiometric data and noise level among workers of Isfehan metal industry. Koomesh. 2009 Aug 15;10(4):253-60.

13. De D. RobinowitzP. Noise in textbook of clinical occupational and environmental medicine. Rosen L, Stock, ED 2nd ed Philadelphia USA: Elsevier saunders. 2005:893.

14. Boman E. Noise in the school environment-Memory and Annoyance (Doctoral dissertation, Byggvetenskap). 2004.

15. Stansfeld SA, Berglund B, Clark C, Lopez-Barrio I, Fischer P, Öhrström E, et al. Aircraft and road traffic noise and children's cognition and health: a cross-national study. The Lancet. 2005;365(9475):1942-9.

16. Buss AH, Perry M. The aggression questionnaire. Journal of personality and social psychology. 1992 Sep;63(3):452.

17. Longo DL, Fauci AS, Kasper DL, Hauser SL, Jameson JL, Loscalzo J. Harrison's principles of internal medicine 18E Vol 2 EB: McGraw Hill Professional; 2012.

18. Alimohammadi I, Nassiri P, Azkhosh M, Hoseini M. Factors affecting road traffic noise annoyance among white-collar employees working in Tehran. Journal of Environmental Health Science \& Engineering. 2010;7(1):2534.

19. Rutherford A, Zwi AB, Grove NJ, Butchart A. Violence: a priority for public health?(part 2). Journal of Epidemiology \& Community Health. 2007 Sep 1;61(9):764-70.

20. Finkel EJ, DeWall CN, Slotter EB, McNulty JK, Pond Jr RS, Atkins DC. Using $\mathrm{I}^{3}$ theory to clarify when dispositional aggressiveness predicts intimate partner violence perpetration.
Journal of personality and social psychology. 2012 Mar;102(3):533.

21. Brouček J. Effect of noise on performance, stress, and behaviour of animals. Slovak journal of animal science. 2014;47(2):111-23.

22. Constantinou E, Panayiotou G, Konstantinou N, Loutsiou-Ladd A, Kapardis A. Risky and aggressive driving in young adults: Personality matters. Accident Analysis \& Prevention. 2011 Jul 1;43(4):1323-31.

23. Saedpanah K, Saedpanah S, Salari S. Investigation of the effects of noise annoyance on the Sleep disturbance among workers of a textile industry. OCCUPATIONAL MEDICINE Quarterly Journal. 2017;9(4):73-82.

24. Pathak V, Tripathi BD, kumar Mishra V. Evaluation of traffic noise pollution and attitudes of exposed individuals in working place. Atmospheric Environment. 2008 May 1;42(16):3892-8.

25. Ferrite S, Santana V. Joint effects of smoking, noise exposure and age on hearing loss. Occupational medicine. 2005 Jan 1;55(1):48-53.

26. Stansfeld SA, Matheson MP. Noise pollution: non-auditory effects on health. British medical bulletin. 2003 Dec 1;68(1):243-57.

27. Lee JH, Kang W, Yaang SR, Choy N, Lee CR. Cohort study for the effect of chronic noise exposure on blood pressure among male workers in Busan, Korea. American journal of industrial medicine. 2009 Jun;52(6):509-17.

28. Fogari R, Zoppi A, Corradi L, Marasi G, Vanasia A, Zanchetti A. Transient but not sustained blood pressure increments by occupational noise. An ambulatory blood pressure measurement study. Journal of Hypertension. 2001 Jun 1;19(6):1021-7.

29. Kalantary S, Dehghani A, Yekaninejad MS, Omidi L, Rahimzadeh M. The effects of occupational noise on blood pressure and heart rate of workers in an automotive parts industry. ARYA atherosclerosis. $2015 \mathrm{Jul} ; 11(4): 215$.

30. Green MS, Schwartz K, Harari G, Najenson $\mathrm{T}$. Industrial noise exposure and ambulatory blood pressure and heart rate. Journal of occupational medicine.: official publication of the Industrial Medical Association. 1991 Aug;33(8):879-83.

31. Babisch W, Pershagen G, Selander J, Houthuijs D, Breugelmans O, Cadum E, Vigna-Taglianti F, Katsouyanni K, Haralabidis AS, Dimakopoulou K, Sourtzi P. Noise annoyance-a modifier of the association between noise level and cardiovascular health?. Science of the total environment. 2013 May 1;452:50-7 


$$
\text { فخرالدين احمدى كانرش و همكاران| بررسى اثرات روانى و فيزيولوزيكى ناشى از مواجهؤ مزمن با صدا.. }
$$

32. Ndrepepa A, Twardella D. Relationship between noise annoyance from road traffic noise and cardiovascular diseases: a meta-analysis. Noise and Health. 2011 May 1;13(52):251. 